\title{
A GEOMETRIC INEQUALITY FOR STABLE SOLUTIONS OF SEMILINEAR ELLIPTIC PROBLEMS IN THE ENGEL GROUP
}

\author{
Andrea Pinamonti and Enrico Valdinoci \\ Università di Trento, Dipartimento di Matematica \\ Via Sommarive 14, I-38123 Povo (TN), Italy; pinamonti@science.unitn.it \\ Università di Milano, Dipartimento di Matematica \\ Via Festa del Perdono 7, I-20122 Milano, Italy; enricovaldinoci@gmail.com
} then

Abstract. We prove that, if $\mathbf{E}$ is the Engel group and $u$ is a stable solution of $\Delta_{\mathbf{E}} u=f(u)$,

$$
\int_{\left\{\nabla_{\mathbf{E}} u \neq 0\right\}}\left[\left|\nabla_{\mathbf{E}} u\right|^{2}\left\{\left(p+\frac{\left\langle(H u)^{T} \nu, v\right\rangle}{\left|\nabla_{\mathbf{E}} u\right|}\right)^{2}+h^{2}\right\}-\mathcal{J}\right] \eta^{2} \leq \int_{\mathbf{E}}\left|\nabla_{\mathbf{E}} \eta\right|^{2}\left|\nabla_{\mathbf{E}} u\right|^{2}
$$

for any test function $\eta \in C_{0}^{\infty}(\mathbf{E})$. Here above, $h$ is the horizontal mean curvature, $p$ is the imaginary curvature and

$$
\mathcal{J}:=2\left(X_{3} X_{2} u X_{1} u-X_{3} X_{1} u X_{2} u\right)+\left(X_{4} u\right)\left(X_{1} u-X_{2} u\right)
$$

This can be interpreted as a geometric Poincaré inequality, extending the work of $[21,22,13]$ to stratified groups of step 3. As an application, we provide a non-existence result.

\section{Introduction}

The Engel group is the Lie group $\mathbf{E}$ having Lie algebra $\mathfrak{e}$ spanned by the vector fields $X_{1}, X_{2}, X_{3}, X_{4}$ subject to the commuting relations

$$
\left[X_{1}, X_{2}\right]=X_{3}, \quad\left[X_{1}, X_{3}\right]=\left[X_{2}, X_{3}\right]=X_{4} .
$$

The sub-Riemannian geometry of $\mathbf{E}$ is the one in which the horizontal vectors in $V_{1}:=\operatorname{span}\left(X_{1}, X_{2}\right)$ play a distinguished role. The geometry is not trivial because $V_{1}, V_{2}:=\left[V_{1}, V_{1}\right]$ and $V_{3}:=\left[V_{1}, V_{2}\right]$ span $\mathfrak{e}$. The essence of sub-Riemannian geometry is that its information is carried out only by $V_{1}$, but in a way which might differ from the Riemannian case. For istance, to better understand the difficulties hidden by the misterious geometry of the Engel group before going into the details of the semilinear equations on it let us consider the case of geodesics. The distance between two points $P$ and $Q$ in $\mathbf{E}$ can be computed as the infimum of the sub-Riemannian length of smooth curves joining them. Namely, we require that $\dot{\gamma}=a_{1} X_{1}+a_{2} X_{2} \in V_{1}$ and compute the length as

$$
\operatorname{length}(\gamma)=\int \sqrt{a_{1}^{2}+a_{2}^{2}} d t
$$

By definition there are $\infty^{1}$ choices for the (unit) tangent vector of a geodesic at a point $P$ of $\mathbf{E}$, but there are $\infty^{4}$ points in $\mathbf{E}$ and (Chow's Theorem) they can all be reached from $P$ along geodesics. Having each geodesic $\infty^{1}$ points, there must 
be two "hidden" real parameters to account for the $\infty^{3}$ multiplicity to be reached starting from each tangent vector at $P$. In particulat, let us consider a smooth threedimensional hypersurface $S \subset \mathbf{E}$ with $P \in \mathbf{E}$ and consider the problem of finding the geodesic starting at $P$ such that, for small $t$, which is the only length minimizing geodesic between $P$ and $\gamma(t)$. Surely, $\dot{\gamma}(t):=\nu \in V_{1}$ must be the vector normal to $S$ in the sub-Riemannian geometry, but this leaves us with $\infty^{2}$ geodesics to choose from. In [1] and [2] the problem was considered in the Heisenberg group, where the only missing parameter was rescued in terms of the "imaginary curvature" of $S$. In [3] the same problem was considered in groups of any step, although most applications were proved in the step-two case only. We refer to Section 2 for a more detailed treatment of the Engel group.

We now introduce the problem we study and present our main result. Given a domain $\Omega \subseteq \mathbf{E}$ we consider solutions $u$ of the following semilinear equation in $\mathbf{E}$,

$$
\Delta_{\mathbf{E}} u=f(u) .
$$

For simplicity, we assume $f \in C^{\infty}(\mathbf{R})$ : in this way, $u \in C^{\infty}(\Omega)$ by the regularity theory of [18] (the case of less regular nonlinearities $f$ may be treated analogously, with only minor modifications).

Moreover, we assume that $u$ is stable, that is

$$
0 \leq \int_{\mathbf{E}}\left\langle\nabla_{\mathbf{E}} \eta, \nabla_{\mathbf{E}} \eta\right\rangle_{\mathbf{E}}+\int_{\mathbf{E}} \dot{f}(u) \eta^{2}
$$

for every $\eta \in C_{0}^{\infty}(\Omega)$.

The stability condition in (2) has been widely studied in the calculus of variation setting: indeed, it states that the second variation of the energy functional associated to (1) is nonnegative at the critical point $u$-hence, for instance, minimal solutions are always stable, but, in principle, stability is a weaker condition than minimality.

Equation (1) is called semilinear, since the only nonlinearity depends on the solution $u$ (not on the space, neither on the derivatives of $u$ ): such kind of equations have been studied in detail in the Euclidean framework, and in the sub-Riemannian one as well (see, e.g. $[5,6,7,17]$ ), and they possess the remarkable geometric property that the operator is constant along the level sets of the solution.

At any point of

we denote by

$$
\mathbf{E}_{0}:=\left\{x \in \Omega \mid \nabla_{\mathbf{E}} u \neq 0\right\}
$$

$$
\nu=\frac{\nabla_{\mathbf{E}} u}{\left|\nabla_{\mathbf{E}} u\right|}
$$

the intrinsic unit normal to the level set of $u$, to wit $\nu$ is the normalized projection on the horizontal fiber of the Riemannian normal.

We shall also consider the intrinsic tangent direction to the level set of $u$

$$
v:=-J \nu,
$$

where $J$ denotes the symplectic matrix

$$
J:=\left(\begin{array}{cc}
0 & -1 \\
1 & 0
\end{array}\right) .
$$

Let us observe that $\forall p \in \mathbf{E}_{0}$

$$
\langle\nu(p), v(p)\rangle_{p, \mathbf{E}}=0,
$$


where $\langle\cdot, \cdot\rangle_{p, \mathbf{E}}$ is the standard scalar product on the fiber $H_{p} \mathbf{E}$. We denote by $H u$ the intrinsic Hessian matrix, i.e.

$$
H u:=\left(\begin{array}{ll}
X_{1} X_{1} u & X_{2} X_{1} u \\
X_{1} X_{2} u & X_{2} X_{2} u
\end{array}\right)
$$

As usual, we define

$$
(H u)^{2}:=(H u)(H u)^{T}
$$

and

$$
|H u|:=\sqrt{\left|\nabla_{\mathbf{E}} X_{1} u\right|^{2}+\left|\nabla_{\mathbf{E}} X_{2} u\right|^{2}} .
$$

Also, in $\mathbf{E}_{0}$, following an analogy in the Heisenberg group (see $[1,2,19,20]$ ), we define the horizontal mean curvature

$$
h:=\operatorname{div}_{\mathbf{E}} \nu
$$

and the imaginary curvature

$$
p:=-\frac{X_{3} u}{\left|\nabla_{\mathbf{E}} u\right|}
$$

Let also

$$
\mathcal{J}:=2\left(X_{3} X_{2} u X_{1} u-X_{3} X_{1} u X_{2} u\right)+\left(X_{4} u\right)\left(X_{1} u-X_{2} u\right) .
$$

With this notation, we have:

\section{Theorem 1.1.}

$$
\int_{\mathbf{E}_{0}}\left[\left|\nabla_{\mathbf{E}} u\right|^{2}\left\{\left(p+\frac{\left\langle(H u)^{T} \nu, v\right\rangle}{\left|\nabla_{\mathbf{E}} u\right|}\right)^{2}+h^{2}\right\}-\mathcal{J}\right] \eta^{2} \leq \int_{\mathbf{E}}\left|\nabla_{\mathbf{E}} \eta\right|^{2}\left|\nabla_{\mathbf{E}} u\right|^{2}
$$

for any $\eta \in C_{0}^{\infty}(\Omega)$.

Theorem 1.1 is a sort of geometric weighted Poincaré inequality, in the sense that the weighted $L^{2}$-norm of any test function is bounded by a weighted $L^{2}$-norm of its gradient, and the weights are built with geometric objects.

In the Euclidean case, the analogue of Theorem 1.1 was established in [21, 22], and recently many extensions have been performed (see, in particular, [11, 12]). As far as we know, the first applications in the sub-Riemannian setting, were performed in $[4,13]$ for the Heisenberg group and in [14] for the Grushin plane. In several cases, these type of geometric weighted inequalities lead to rigidity results (such as classification, symmetry, or non existence, of solutions). Differently from the Euclidean case, the weight on the left hand side of the inequality does not need to be positive in general, due to the presence of $\mathcal{J}$. Thus, the presence of noncommutating vector fields, complicates the geometry of the level sets via the sign of $\mathcal{J}$. Indeed, if $\mathcal{J} \leq 0$, when the right hand side of the inequality in Theorem 1.1 vanishes, one obtains that the level sets of $u$ satisfy the geometric equations, see Corollary 4.2,

$$
\left\{\begin{array}{l}
p+\frac{\left\langle(H u)^{T} \nu, v\right\rangle}{\left|\nabla_{\mathbf{E}} u\right|}=0 \\
h=0
\end{array}\right.
$$

Also, a more geometric interpretation of the quantity $\mathcal{J}$, in dependence of the intrinsic tangent and normal vectors, will be given in Lemma 3.8. 
In this paper, Theorem 1.1 is a first attempt to adapt these geometric weighted inequalities to stratified groups of step higher than 2 (for other different weighted inequalities in $\mathbf{R}^{4}$ obtained via the Engel group, see Theorem 1.4 of [15]).

The higher the step of the group, the more complicated are the combinatorics occurring in the inequality, and the more difficult is the geometric interpretation of the quantities involved. Nevertheless, the Engel group still mantains a reasonable level of geometric insight and provides a challenging source of problems for this approach. For instance, we think that it would be interesting to investigate whether or not rigidity results and geometric properties of stable solutions may be obtained from these kind of Poincaré inequalities (or by other methods as well). In this spirit we are able to prove a first non-existence result for semilinear equations in the Engel group in Theorem 4.7 .

To conclude this introduction we think that is interesting to propose some suggestion for further research. In particular we think that it could be interesting to see if a result like the one proved in Theorem 1.1 holds in more general Carnot groups. Moreover, we think that it could be stimulating to study the geometric nature of $\mathcal{J}$, in particular it would be desirable to relate $\mathcal{J}$ with the sub-Riemannian metric structure of $\mathbf{E}$.

The organization of this paper is as follows. In Section 2, we recall what the Engel group is and what its basic properties are. Then, we prove Theorem 1.1 in Section 3. Finally, in Section 4, we derive the geometric equations (6) in Corollary 4.2, and we provide a non-existence result in Theorem 4.7.

\section{The Engel group}

We recall the basic definitions and properties of the Engel group.

Definition 2.1. The Engel algebra is the finite dimensional Lie algebra $\mathfrak{e}$ with basis $\left(X_{1}, X_{2}, X_{3}, X_{4}\right)$ where the only nonvanishing commutators relationship among the generators are

$$
\left[X_{1}, X_{2}\right]=X_{3}, \quad\left[X_{1}, X_{3}\right]=\left[X_{2}, X_{3}\right]=X_{4} .
$$

Remark 2.2. It is easy to see that the Engel algebra is stratified of step 3, i.e.

$$
\mathfrak{e}=\mathfrak{e}_{1} \oplus \mathfrak{e}_{2} \oplus \mathfrak{e}_{3},
$$

where $\mathfrak{e}_{1}:=\operatorname{span}\left\{X_{1}, X_{2}\right\}, \mathfrak{e}_{2}:=\operatorname{span}\left\{X_{3}\right\}$ and $\mathfrak{e}_{3}:=\operatorname{span}\left\{X_{4}\right\}$ and

$$
\left[\mathfrak{e}_{1}, \mathfrak{e}_{1}\right]=\mathfrak{e}_{2}, \quad\left[\mathfrak{e}_{1}, \mathfrak{e}_{2}\right]=\mathfrak{e}_{3}, \quad\left[\mathfrak{e}_{1}, \mathfrak{e}_{3}\right]=\{0\} .
$$

Definition 2.3. The Engel group, denoted by $\mathbf{E}$, is the simply connected nilpotent Lie group associated to $\mathfrak{e}$.

Since $\mathbf{E}$ is a Carnot group we can represent it by means of graded coordinates associated to the basis $\left(X_{1}, X_{2}, X_{3}, X_{4}\right)$; it follows that $\mathbf{E}=\left(\mathbf{R}^{4}, \cdot,\left\{\delta_{r}\right\}_{\{r>0\}}\right)$, where $\forall\left(x_{1}, x_{2}, x_{3}, x_{4}\right),\left(y_{1}, y_{2}, y_{3}, y_{4}\right) \in \mathbf{E}$,

$$
\begin{aligned}
\left(x_{1}, x_{2}, x_{3}, x_{4}\right) \cdot\left(y_{1}, y_{2}, y_{3}, y_{4}\right):= & \left(x_{1}+y_{1}, x_{2}+y_{2}, x_{3}+y_{3}+\frac{1}{2}\left(x_{1} y_{2}-x_{2} y_{1}\right),\right. \\
& x_{4}+y_{4}+\frac{1}{2}\left[\left(x_{1} y_{3}-x_{3} y_{1}\right)+\left(x_{2} y_{3}-x_{3} y_{2}\right)\right] \\
& \left.+\frac{1}{12}\left[\left(x_{1}-y_{1}+x_{2}-y_{2}\right)\left(x_{1} y_{2}-x_{2} y_{1}\right)\right]\right)
\end{aligned}
$$


and the homogeneous dilations on $\mathbf{E}$ are

$$
\delta_{r}\left(x_{1}, x_{2}, x_{3}, x_{4}\right):=\left(r x_{1}, r x_{2}, r^{2} x_{3}, r^{3} x_{4}\right), \quad r>0 .
$$

The rappresentation of the basis $\left(X_{1}, X_{2}, X_{3}, X_{4}\right)$ on the graded coordinates gives

$$
\begin{aligned}
& X_{1}\left(x_{1}, x_{2}, x_{3}, x_{4}\right)=\partial_{1}-\frac{x_{2}}{2} \partial_{3}-\left(\frac{x_{3}}{2}+\frac{x_{2}}{12}\left(x_{1}+x_{2}\right)\right) \partial_{4}, \\
& X_{2}\left(x_{1}, x_{2}, x_{3}, x_{4}\right)=\partial_{2}+\frac{x_{1}}{2} \partial_{3}-\left(\frac{x_{3}}{2}-\frac{x_{1}}{12}\left(x_{1}+x_{2}\right)\right) \partial_{4}, \\
& X_{3}\left(x_{1}, x_{2}, x_{3}, x_{4}\right)=\partial_{3}+\frac{1}{2}\left(x_{1}+x_{2}\right) \partial_{4}, \\
& X_{4}\left(x_{1}, x_{2}, x_{3}, x_{4}\right)=\partial_{4} .
\end{aligned}
$$

We fix a left invariant Riemannian metric on $\mathbf{R}^{4}$ that makes the above vector fields orthonormal, i.e. at every $p \in \mathbf{E}$ we give a scalar product $\langle\cdot, \cdot\rangle_{p, \mathbf{E}}$ such that

$$
\left\langle X_{i}(p), X_{i}(p)\right\rangle_{p, \mathbf{E}}=1, \quad i \in\{1,2,3,4\},
$$

and

$$
\left\langle X_{i}(p), X_{j}(p)\right\rangle_{p, \mathbf{E}}=0 \quad \text { if } i \neq j .
$$

As usual in the context of Carnot groups, for every $p \in \mathbf{E}$ we define the horizontal fiber at $p$ as the subspace of the tangent space at $p$ generated by $X_{1}(p)$ and $X_{2}(p)$, i.e.

$$
H_{p} \mathbf{E}:=\operatorname{span}\left\{X_{1}(p), X_{2}(p)\right\} \subset T_{p} \mathbf{E}
$$

and the horizontal subbundle of the tangent boundle associated to $\mathbf{E}$ as

$$
H \mathbf{E}:=\bigcup_{p \in \mathbf{E}} H_{p} \mathbf{E} .
$$

Finally, we briefly recall the notion of Carnot-Carathéodory distance on $\mathbf{E}$, see [7] for a more detailed treatment.

Definition 2.4. A locally Lipschitz curve $\lambda:[0, T] \rightarrow \mathbf{R}^{4}$ is said to be horizontal if there are $c_{1}, c_{2} \in L^{\infty}([0, T])$ such that

$$
\dot{\lambda}(t)=c_{1}(t) X_{1}(\lambda(t))+c_{2}(t) X_{2}(\lambda(t)) \quad \mathcal{L}^{1} \text {-q.o. } t \in[0, T]
$$

and

$$
c_{1}(t)^{2}+c_{2}(t)^{2} \leq 1 \quad \text { in }[0, T] .
$$

The CC-distance between two points $p, q \in \mathbf{E}$ is defined as follows

$$
\mathrm{d}_{\mathrm{CC}}(x, y):=\inf \left\{T>0 \mid \exists \lambda:[0, T] \longrightarrow \mathbf{R}^{4} \text { horizontal s.t. } \lambda(0)=x, \lambda(T)=y\right\} .
$$

Remark 2.5. It is a classical result that $\mathrm{d}_{C C}$ is a distance on $\mathbf{E}$, see [9]. Moreover, we have

$$
\mathrm{d}_{\mathrm{CC}}(x, y)=\mathrm{d}_{\mathrm{CC}}(z \cdot x, z \cdot y) \quad \forall x, y, z \in \mathbf{E}
$$

and

$$
\mathrm{d}_{\mathrm{CC}}\left(\delta_{r}(x), \delta_{r}(y)\right)=r \mathrm{~d}_{\mathrm{CC}}(x, y) \quad \forall x, y \in \mathbf{E}, \forall r \in(0, \infty) .
$$

Finally, let $K \Subset \Omega$ be a compact set. Then there exists a constant $\alpha>0$ such that

$$
\mathrm{d}_{\mathrm{CC}}(x, y) \geq \alpha|x-y|
$$

for all $x, y \in K$. 
Definition 2.6. Let $u: \Omega \rightarrow \mathbf{R}$ be a $C^{1}$ map then the horizontal gradient of $u$ is defined as follows

$$
\nabla_{\mathbf{E}} u(x):=X_{1} u(x) X_{1}+X_{2} u(x) X_{2} .
$$

Moreover, if $u \in C^{1}\left(\Omega, \mathbf{R}^{2}\right)$, then the horizontal divergence of $u$ is

$$
\operatorname{div}_{\mathbf{E}} u(x):=X_{1} u_{1}(x)+X_{2} u_{2}(x)
$$

Finally, if $u: \Omega \longrightarrow \mathbf{R}$ is $C^{2}$, then the horizontal Laplacian of $u$ is

$$
\Delta_{\mathbf{E}} u(x):=X_{1} X_{1} u(x)+X_{2} X_{2} u(x) .
$$

Remark 2.7. Let us explicitely observe that the intrinsic gradient does not depend of the basis $\left(X_{1}, X_{2}\right)$.

Remark 2.8. If, in Theorem 1.1, $u$ does not depend on $x_{4}$, then the situation boils down to the one in the Heisenberg group (note indeed that $X_{3} X_{1} u=X_{1} X_{3} u$, so Theorem 1.1 reduces to Theorem 2.3 in [13]).

\section{Proof of Theorem 1.1}

The proof of our first result needs some preliminary, technical computations, by which we obtain some useful identities.

Lemma 3.1. Let $j \in\{1,2\}$. If $u \in C^{\infty}(\Omega)$, then in $\mathbf{E}_{0}$ we have

$$
X_{j}\left|\nabla_{\mathbf{E}} u\right|=\left\langle X_{j}\left(\nabla_{\mathbf{E}} u\right), \nu\right\rangle_{\mathbf{E}}
$$

Moreover, for each $\eta \in C_{0}^{\infty}(\Omega)$,

$$
\nabla_{\mathbf{E}}\left(\left|\nabla_{\mathbf{E}} u\right| \eta\right)=\frac{\eta}{\left|\nabla_{\mathbf{E}} u\right|}(H u)^{T} \nabla_{\mathbf{E}} u+\left|\nabla_{\mathbf{E}} u\right| \nabla_{\mathbf{E}} \eta
$$

and

$$
\left|\nabla_{\mathbf{E}}\left(\left|\nabla_{\mathbf{E}} u\right| \eta\right)\right|^{2}=\frac{\eta^{2}}{\left|\nabla_{\mathbf{E}} u\right|^{2}}\left|(H u)^{T} \nabla_{\mathbf{E}} u\right|^{2}+2 \eta\left\langle\nabla_{\mathbf{E}} u,(H u) \nabla_{\mathbf{E}} \eta\right\rangle_{\mathbf{E}}+\left|\nabla_{\mathbf{E}} \eta\right|^{2}\left|\nabla_{\mathbf{E}} u\right|^{2}
$$

Proof. Equation (11) is straightforward. Also, the proof of (12) follows from the following simple calculation:

$$
\nabla_{\mathbf{E}}\left(\left|\nabla_{\mathbf{E}} u\right| \eta\right)=\eta \nabla_{\mathbf{E}}\left(\left|\nabla_{\mathbf{E}} u\right|\right)+\left|\nabla_{\mathbf{E}} u\right| \nabla_{\mathbf{E}} \eta=\frac{\eta}{\left|\nabla_{\mathbf{E}} u\right|}(H u)^{T} \nabla_{\mathbf{E}} u+\left|\nabla_{\mathbf{E}} u\right| \nabla_{\mathbf{E}} \eta
$$

Furthermore,

$$
\begin{aligned}
\left|\nabla_{\mathbf{E}}\left(\left|\nabla_{\mathbf{E}} u\right| \eta\right)\right|^{2}= & \left\langle\nabla_{\mathbf{E}}\left(\left|\nabla_{\mathbf{E}} u\right| \eta\right), \nabla_{\mathbf{E}}\left(\left|\nabla_{\mathbf{E}} u\right| \eta\right)\right\rangle_{\mathbf{E}} \\
= & \left\langle\frac{\eta}{\left|\nabla_{\mathbf{E}} u\right|}(H u)^{T} \nabla_{\mathbf{E}} u, \frac{\eta}{\left|\nabla_{\mathbf{E}} u\right|}(H u)^{T} \nabla_{\mathbf{E}} u\right\rangle_{\mathbf{E}} \\
& +2\left\langle\frac{\eta}{\left|\nabla_{\mathbf{E}} u\right|}(H u)^{T} \nabla_{\mathbf{E}} u,\left|\nabla_{\mathbf{E}} u\right| \nabla_{\mathbf{E}} \eta\right\rangle_{\mathbf{E}}+\left\langle\left|\nabla_{\mathbf{E}} u\right| \nabla_{\mathbf{E}} \eta,\left|\nabla_{\mathbf{E}} u\right| \nabla_{\mathbf{E}} \eta\right\rangle_{\mathbf{E}}
\end{aligned}
$$

Hence

$$
\begin{aligned}
\left|\nabla_{\mathbf{E}}\left(\left|\nabla_{\mathbf{E}} u\right| \eta\right)\right|^{2} & =\left(\frac{\eta}{\left|\nabla_{\mathbf{E}} u\right|}\right)^{2}\left|(H u)^{T} \nabla_{\mathbf{E}} u\right|^{2}+2 \eta\left\langle(H u)^{T} \nabla_{\mathbf{E}} u, \nabla_{\mathbf{E}} \eta\right\rangle_{\mathbf{E}}+\left|\nabla_{\mathbf{E}} \eta\right|^{2}\left|\nabla_{\mathbf{E}} u\right|^{2} \\
& =\left(\frac{\eta}{\left|\nabla_{\mathbf{E}} u\right|}\right)^{2}\left|(H u)^{T} \nabla_{\mathbf{E}} u\right|^{2}+2 \eta\left\langle\nabla_{\mathbf{E}} u,(H u) \nabla_{\mathbf{E}} \eta\right\rangle_{\mathbf{E}}+\left|\nabla_{\mathbf{E}} \eta\right|^{2}\left|\nabla_{\mathbf{E}} u\right|^{2}
\end{aligned}
$$

and this proves (13). 
Lemma 3.2. Let $u \in C^{\infty}(\Omega)$. Then

$$
|H u|^{2}-\left\langle(H u)^{2} \nu, \nu\right\rangle_{\mathbf{E}}=\left|(H u)^{T} v\right|^{2} .
$$

Moreover, in $\mathbf{E}_{0}$

$$
\left|(H u)^{T} v\right|^{2}=\left|\nabla_{\mathbf{E}} u\right|^{2}\left\{\left(p+\frac{\left\langle(H u)^{T} \nu, v\right\rangle}{\left|\nabla_{\mathbf{E}} u\right|}\right)^{2}+h^{2}\right\} .
$$

Proof. We note that for each $q \in \mathbf{E}(\nu(q), v(q))$ is an orthonormal basis of $H_{q} \mathbf{E}$. Then (15) follows, for instance, from Lemma 3 in [4].

In order to prove (16), we begin observing that

$$
\begin{aligned}
& (H u)^{T}=\left(\begin{array}{ll}
X_{1} X_{1} u & X_{1} X_{2} u \\
X_{2} X_{1} u & X_{2} X_{2} u
\end{array}\right) \\
& =\left(\begin{array}{ll}
X_{1} X_{1} u & X_{2} X_{1} u \\
X_{1} X_{2} u & X_{2} X_{2} u
\end{array}\right)+\left(\begin{array}{cc}
0 & X_{1} X_{2} u-X_{2} X_{1} u \\
X_{2} X_{1} u-X_{1} X_{2} u & 0
\end{array}\right) \\
& =H u+\left(\begin{array}{cc}
0 & X_{3} u \\
-X_{3} u & 0
\end{array}\right) .
\end{aligned}
$$

Let also $\mathcal{Z}$ and $H_{\nu} \in \operatorname{Mat}(\mathbf{R}, 2 \times 2)$ be defined as

$$
\mathcal{Z}:=\nu \otimes(H u)^{T} \nu \text { and }\left(H_{\nu}\right)_{i j}:=X_{j}\left(\nu_{i}\right)
$$

for $i, j \in\{1,2\}$. So, we use (11) to obtain that

$$
\begin{aligned}
\mathcal{Z}_{i j}+\left|\nabla_{\mathbf{E}} u\right|\left(H_{\nu}\right)_{i j} & =\nu_{i}\left\langle X_{j}\left(\nabla_{\mathbf{E}} u\right), \nu\right\rangle_{\mathbf{E}}+\left|\nabla_{\mathbf{E}} u\right| X_{j}\left(\frac{X_{i} u}{\left|\nabla_{\mathbf{E}} u\right|}\right) \\
& =\frac{X_{i} u}{\left|\nabla_{\mathbf{E}} u\right|}\left\langle X_{j}\left(\nabla_{\mathbf{E}} u\right), \nu\right\rangle_{\mathbf{E}}+X_{j} X_{i} u-\frac{X_{i} u}{\left|\nabla_{\mathbf{E}} u\right|} X_{j}\left|\nabla_{\mathbf{E}} u\right|=X_{j} X_{i} u,
\end{aligned}
$$

that is,

$$
\mathcal{Z}+\left|\nabla_{\mathbf{E}} u\right| H_{\nu}=H u
$$

Hence, we can rewrite (17) in the following way

$$
(H u)^{T}=\left(X_{3} u\right) J+\mathcal{Z}+\left|\nabla_{\mathbf{E}} u\right| H_{\nu}
$$

Furthermore, we immediately get:

$$
\mathcal{Z} v=\left\langle(H u)^{T} \nu, v\right\rangle_{\mathbf{E}} \nu
$$

By plugging (19) into (18), we conclude that

$$
(H u)^{T} v=\left(-X_{3} u+\left\langle(H u)^{T} \nu, v\right\rangle_{\mathbf{E}}\right) \nu+\left|\nabla_{\mathbf{E}} u\right| H_{\nu} v
$$

and so

$$
\begin{aligned}
\left|(H u)^{T} v\right|^{2}= & \left\langle(H u)^{T} v,(H u)^{T} v\right\rangle_{\mathbf{E}}=\left(-X_{3} u+\left\langle(H u)^{T} \nu, v\right\rangle_{\mathbf{E}}\right)^{2}+\left|\nabla_{\mathbf{E}} u\right|^{2}\left|H_{\nu} v\right|^{2} \\
& +2\left(-X_{3} u+\left\langle(H u)^{T} \nu, v\right\rangle_{\mathbf{E}}\right)\left|\nabla_{\mathbf{E}} u\right|\left\langle H_{\nu} v, \nu\right\rangle_{\mathbf{E}} .
\end{aligned}
$$

From this and the definitions in (4) and (5), we obtain that the proof of (16) is completed if we prove that

$$
\left\langle H_{\nu} v, \nu\right\rangle_{\mathbf{E}}=0
$$

and that

$$
\left|H_{\nu} v\right|=\left|\operatorname{div}_{\mathbf{E}} \nu\right| .
$$


To this end, let us observe that, by (20),

$$
\left|\nabla_{\mathbf{E}} u\right|\left\langle H_{\nu} v, \nu\right\rangle_{\mathbf{E}}=\left\langle(H u)^{T} v, \nu\right\rangle_{\mathbf{E}}-\langle(H u) v, \nu\rangle_{\mathbf{E}}+X_{3} u .
$$

Now, by (17),

$$
(H u)^{T} v-(H u) v=\left(\begin{array}{c}
-\frac{X_{1} u}{\left|\nabla_{\mathbf{E}} u\right|} X_{3} u \\
-\frac{X_{2} u}{\left|\nabla_{\mathbf{E}} u\right|} X_{3} u
\end{array}\right)=-\left(X_{3} u\right) \nu
$$

hence

$$
\left\langle(H u)^{T} v-(H u) v, \nu\right\rangle_{\mathbf{E}}=-X_{3} u
$$

By plugging this into (23), we obtain (21).

To obtain (22), we argue as follows. By (21), we know that $v$ is an eigenvector for $H_{\nu} v$ and then

$$
H_{\nu} v=\lambda v
$$

with $|\lambda|=\left|H_{\nu} v\right|$. Hence, by (20),

$$
\pm\left|\nabla_{\mathbf{E}} u\right|\left|H_{\nu} v\right|=\left\langle\left|\nabla_{\mathbf{E}} u\right| H_{\nu} v, v\right\rangle_{\mathbf{E}}=\left\langle(H u)^{T} v, v\right\rangle_{\mathbf{E}}=\sum_{i, j=1}^{2}\left(X_{i} X_{j} u\right) v_{i} v_{j} .
$$

Now, we remark that

$$
\nu_{i}^{2}=1-v_{i}^{2}
$$

To prove this, we take $i=1$ (the case $i=2$ being analogous), and we observe that

$$
\nu_{1}^{2}=v_{2}^{2}=1-v_{1}^{2},
$$

which establishes (25).

On the other hand, if $i \neq j$,

$$
\nu_{i} \nu_{j}=\nu_{1} \nu_{2}=\left(-v_{2}\right)\left(v_{1}\right)=-v_{i} v_{j}
$$

So, by (11), (25) and (26), we obtain

$$
\begin{aligned}
\left|\nabla_{\mathbf{E}} u\right|\left|\operatorname{div}_{\mathbf{E}} \nu\right| & =\left|\nabla_{\mathbf{E}} u\right| \sum_{i=1}^{2} X_{i}\left(\frac{X_{i} u}{\left|\nabla_{\mathbf{E}} u\right|}\right)=\sum_{i=1}^{2} X_{i} X_{i} u-\frac{X_{i} u}{\left|\nabla_{\mathbf{E}} u\right|}\left\langle X_{i}\left(\nabla_{\mathbf{E}} u\right), \nu\right\rangle_{\mathbf{E}} \\
& =\sum_{i=1}^{2} X_{i} X_{i} u-\sum_{i, j=1}^{2}\left(X_{i} X_{j} u\right) \nu_{i} \nu_{j} \\
& =\sum_{i=1}^{2} X_{i} X_{i} u-\sum_{i=1}^{2}\left(X_{i} X_{i} u\right) \nu_{i}^{2}-\sum_{i \neq j=1}^{2}\left(X_{i} X_{j} u\right) \nu_{i} \nu_{j} \\
& =\sum_{i=1}^{2} X_{i} X_{i} u-\sum_{i=1}^{2}\left(X_{i} X_{i} u\right)\left(1-v_{i}^{2}\right)+\sum_{i \neq j=1}^{2}\left(X_{i} X_{j} u\right) v_{i} v_{j} \\
& =\sum_{i=1}^{2}\left(X_{i} X_{i} u\right) v_{i}^{2}+\sum_{i \neq j=1}^{2}\left(X_{i} X_{j} u\right) v_{i} v_{j}=\sum_{i, j=1}^{2}\left(X_{i} X_{j} u\right) v_{i} v_{j} .
\end{aligned}
$$


By comparing (24) and (27), we see that

$$
\left|\nabla_{\mathbf{E}} u\right|\left|H_{\nu} v\right|=\left|\nabla_{\mathbf{E}} u\right|\left|\operatorname{div}_{\mathbf{E}} \nu\right|,
$$

which implies (22), as desired.

Lemma 3.3. For each $u \in C^{\infty}(\Omega)$ it holds that

$$
\begin{aligned}
& X_{1} \Delta_{\mathbf{E}} u=\Delta_{\mathbf{E}} X_{1} u+2 X_{3} X_{2} u+X_{4} u, \\
& X_{2} \Delta_{\mathbf{E}} u=\Delta_{\mathbf{E}} X_{2} u-2 X_{1} X_{3} u+X_{4} u, \\
& X_{3} \Delta_{\mathbf{E}} u=\Delta_{\mathbf{E}} X_{3} u-2 X_{4} X_{1} u-2 X_{4} X_{2} u, \text { and } \\
& X_{4} \Delta_{\mathbf{E}} u=\Delta_{\mathbf{E}} X_{4} u .
\end{aligned}
$$

Proof. For the first equality

$$
\begin{aligned}
X_{1} \Delta_{\mathbf{E}} u & =X_{1}\left(X_{1} X_{1} u\right)+X_{1}\left(X_{2} X_{2} u\right)=\Delta_{\mathbf{E}} X_{1} u+X_{1} X_{2} X_{2} u-X_{2} X_{2} X_{1} u \\
& =\Delta_{\mathbf{E}} X_{1} u+X_{3} X_{2} u+X_{2} X_{1} X_{2} u-X_{2} X_{2} X_{1} u \\
& =\Delta_{\mathbf{E}} X_{1} u+2 X_{3} X_{2} u+X_{2} X_{3} u-X_{3} X_{2} u=\Delta_{\mathbf{E}} X_{1} u+2 X_{3} X_{2} u+X_{4} u .
\end{aligned}
$$

The second and the third equality follow in a similar way, indeed

$$
\begin{aligned}
X_{2} \Delta_{\mathbf{E}} u & =X_{2}\left(X_{1} X_{1} u\right)+X_{2}\left(X_{2} X_{2} u\right)=\Delta_{\mathbf{E}} X_{2} u-X_{1} X_{1} X_{2} u+X_{2} X_{1} X_{1} u \\
& =\Delta_{\mathbf{E}} X_{2} u-X_{3} X_{1} u+X_{1} X_{2} X_{1} u-X_{1} X_{1} X_{2} u=\Delta_{\mathbf{E}} X_{2} u-X_{3} X_{1} u-X_{1} X_{3} u \\
& =\Delta_{\mathbf{E}} X_{2} u-X_{3} X_{1} u+X_{1} X_{3} u-2 X_{1} X_{3} u=\Delta_{\mathbf{E}} X_{2} u-2 X_{1} X_{3} u+X_{4} u
\end{aligned}
$$

and

$$
\begin{aligned}
X_{3} \Delta_{\mathbf{E}} u & =X_{3}\left(X_{1} X_{1} u\right)+X_{3}\left(X_{2} X_{2} u\right)=X_{1} X_{3} X_{1} u+X_{2} X_{3} X_{2} u-X_{4} X_{1} u-X_{4} X_{2} u \\
& =\Delta_{\mathbf{E}} X_{3} u-2 X_{4} X_{1} u-2 X_{4} X_{2} u .
\end{aligned}
$$

The last is a direct consequence of $X_{1} X_{4} u=X_{4} X_{1} u$ and $X_{2} X_{4} u=X_{4} X_{2} u$.

Using Lemma 3.3, we obtain

Corollary 3.4. Let $u \in C^{\infty}(\Omega)$ be a solution of (1). Then

$$
\begin{aligned}
\Delta_{\mathbf{E}} X_{1} u+2 X_{3} X_{2} u+X_{4} u & =\dot{f}(u) X_{1} u, \\
\Delta_{\mathbf{E}} X_{2} u-2 X_{1} X_{3} u+X_{4} u & =\dot{f}(u) X_{2} u, \\
\Delta_{\mathbf{E}} X_{3} u-2 X_{4} X_{1} u-2 X_{4} X_{2} u & =\dot{f}(u) X_{3} u .
\end{aligned}
$$

Now, some observation related to the Coarea formula, in order to reduce the computations in the whole of $\mathbf{E}$ to the one in $\mathbf{E}_{0}$.

Lemma 3.5. If $u: \Omega \rightarrow \mathbf{R}$ is Lipschitz with respect to the $\mathrm{d}_{C C}$ distance, then for every $c \in \mathbf{R}$, the set $\left\{x \in \Omega \mid \nabla_{\mathbf{E}} u(x) \neq 0\right\} \cap\{x \in \Omega \mid u(x)=c\}$ has zero Lebesgue measure.

Proof. For every $f \in L^{1}(\Omega)$ by the Coarea formula proved in [19] we have

$$
\int_{\Omega} f\left|\nabla_{\mathbf{E}} u\right| \mathrm{d} x=\int_{-\infty}^{+\infty}\left(\int_{\{x \in \Omega \mid u(x)=t\}} f \mathrm{~d}\left|\partial E_{t}\right|_{\mathbf{E}}\right) \mathrm{d} t
$$

where $\left|\partial E_{t}\right|_{\mathbf{E}}$ is the Engel group perimeter (see $\left.[7,16,17]\right)$. 
If we take as $f$ the characteristic function of the set $U \cap\{u=c\}$ where $U \subseteq \Omega$ is a bounded domain, then

$$
\int_{\{x \in \Omega \mid u(x)=t\}} f \mathrm{~d}\left|\partial E_{t}\right|_{\mathbf{E}}=0 \quad \forall t \neq c .
$$

Hence

$$
\int_{U \cap\{u=c\}}\left|\nabla_{\mathbf{E}} u\right| \mathrm{d} x=0
$$

that implies the desired result.

Using Lemma 3.5 and (10) it easily follows the following

Corollary 3.6. If $u \in \operatorname{Lip}_{\text {loc }}(\Omega)$, then for every $c \in \mathbf{R}$, the set $\left\{x \in \Omega \mid \nabla_{\mathbf{E}} u(x) \neq\right.$ $0\} \cap\{x \in \Omega \mid u(x)=c\}$ has zero Lebesgue measure.

With this, we are in the position of proving the following geometric inequality:

Proposition 3.7. Let $u \in C^{\infty}(\Omega)$ be a stable weak solution of (1). Then, for each $\eta \in C_{0}^{\infty}(\Omega)$,

$$
\begin{aligned}
& \int_{\mathbf{E}_{0}}\left[|H u|^{2}-\left\langle(H u)^{2} \nu, \nu\right\rangle_{\mathbf{E}}\right] \eta^{2}-2 \int_{\mathbf{E}_{0}}\left(X_{3} X_{2} u X_{1} u-X_{3} X_{1} u X_{2} u\right) \eta^{2} \\
& -\int_{\mathbf{E}_{0}}\left(X_{4} u\right)\left(X_{1} u-X_{2} u\right) \eta^{2} \leq \int_{\mathbf{E}}\left|\nabla_{\mathbf{E}} \eta\right|^{2}\left|\nabla_{\mathbf{E}} u\right|^{2} .
\end{aligned}
$$

Proof. Multiplying by $\left(X_{1} u\right) \eta^{2}$ equation (28) in Corollary 3.4 and by $\left(X_{2} u\right) \eta^{2}$ equation (29) and then integrating by parts we obtain

$$
\begin{aligned}
& -\int_{\mathbf{E}}\left\langle\nabla_{\mathbf{E}} X_{1} u, \nabla_{\mathbf{E}}\left(X_{1} u \eta^{2}\right)\right\rangle_{\mathbf{E}}+2 \int_{\mathbf{E}} X_{3} X_{2} u\left(X_{1} u\right) \eta^{2}+\int_{\mathbf{E}} X_{4} u\left(X_{1} u\right) \eta^{2} \\
& =\int_{\mathbf{E}} \dot{f}(u)\left(X_{1} u\right)^{2} \eta^{2}-\int_{\mathbf{E}}\left\langle\nabla_{\mathbf{E}} X_{2} u, \nabla_{\mathbf{E}}\left(X_{2} u \eta^{2}\right)\right\rangle_{\mathbf{E}}-2 \int_{\mathbf{E}} X_{1} X_{3} u\left(X_{2} u\right) \eta^{2} \\
& \quad+\int_{\mathbf{E}} X_{4} u\left(X_{2} u\right) \eta^{2}=\int_{\mathbf{E}} \dot{f}(u)\left(X_{2} u\right)^{2} \eta^{2} .
\end{aligned}
$$

Consequently, by summing term by term, we get

$$
\begin{aligned}
& -\int_{\mathbf{E}}\left(\left|\nabla_{\mathbf{E}} X_{1} u\right|^{2}+\left|\nabla_{\mathbf{E}} X_{2} u\right|^{2}\right) \eta^{2}-\int_{\mathbf{E}}\left\langle\nabla_{\mathbf{E}} X_{1} u, \nabla_{\mathbf{E}} \eta^{2}\right\rangle_{\mathbf{E}} X_{1} u \\
& -\int_{\mathbf{E}}\left\langle\nabla_{\mathbf{E}} X_{2} u, \nabla_{\mathbf{E}} \eta^{2}\right\rangle_{\mathbf{E}} X_{2} u+2 \int_{\mathbf{E}}\left(X_{3} X_{2} u X_{1} u-X_{1} X_{3} u X_{2} u\right) \eta^{2} \\
& +\int_{\mathbf{E}}\left(X_{4} u\right)\left(X_{1} u+X_{2} u\right) \eta^{2}=\int_{\mathbf{E}} \dot{f}(u)\left|\nabla_{\mathbf{E}} u\right|^{2} \eta^{2} .
\end{aligned}
$$

On the other hand, since $u$ is stable, by choosing $\left|\nabla_{\mathbf{E}} u\right| \eta$ as a test function in (2) we obtain

$$
0 \leq \int_{\mathbf{E}}\left|\nabla_{\mathbf{E}}\left(\left|\nabla_{\mathbf{E}} u\right| \eta\right)\right|^{2}+\int_{\mathbf{E}} \dot{f}(u)\left|\nabla_{\mathbf{E}} u\right|^{2} \eta^{2}
$$


By Corollary 3.6 we have that $\nabla_{\mathbf{E}}\left(\left|\nabla_{\mathbf{E}} u\right| \eta\right)=0$ almost everywhere outside $\mathbf{E}_{0}$; hence making use of (13) we obtain from (34) that

$$
\begin{aligned}
0 \leq & \int_{\mathbf{E}_{0}}\left(\frac{\eta^{2}}{\left|\nabla_{\mathbf{E}} u\right|^{2}}\left|(H u)^{T} \nabla_{\mathbf{E}} u\right|^{2}+2 \eta\left\langle(H u)^{T} \nabla_{\mathbf{E}} u, \nabla_{\mathbf{E}} \eta\right\rangle_{\mathbf{E}}+\left|\nabla_{\mathbf{E}} \eta\right|^{2}\left|\nabla_{\mathbf{E}} u\right|^{2}\right) \\
& +\int_{\mathbf{E}} \dot{f}(u)\left|\nabla_{\mathbf{E}} u\right|^{2} \eta^{2} .
\end{aligned}
$$

So, noticing that $2 \eta \nabla_{\mathbf{E}} \eta=\nabla_{\mathbf{E}} \eta^{2}$, and using (32), after a simplification we obtain that

$$
\begin{aligned}
& \int_{\mathbf{E}}|H u|^{2} \eta^{2}-\int_{\mathbf{E}_{0}} \frac{\eta^{2}}{\left|\nabla_{\mathbf{E}} u\right|^{2}}\left|(H u)^{T} \nabla_{\mathbf{E}} u\right|^{2}-2 \int_{\mathbf{E}}\left(X_{3} X_{2} u X_{1} u-X_{1} X_{3} u X_{2} u\right) \eta^{2} \\
& -\int_{\mathbf{E}}\left(X_{4} u\right)\left(X_{1} u+X_{2} u\right) \eta^{2} \leq \int_{\mathbf{E}_{0}}\left|\nabla_{\mathbf{E}} \eta\right|^{2}\left|\nabla_{\mathbf{E}} u\right|^{2} .
\end{aligned}
$$

Recalling that

$$
X_{1} X_{3} u=X_{3} X_{1} u+X_{4} u
$$

we get the thesis.

Then, from Proposition 3.7 and Lemma 3.2 we immediately obtain Theorem 1.1.

We end this section by giving some more geometric insight on the quantity $\mathcal{J}$, in relation with the intrinsic normal and tangent vectors:

Lemma 3.8. For every $u \in C^{2}(\Omega)$ and every $x \in \mathbf{E}_{0}$ it holds

$$
\mathcal{J}(x)=-\left|\nabla_{\mathbf{E}} u\right|(x)\left\langle\nabla_{\mathbf{E}} X_{3} u(x), v(x)\right\rangle_{\mathbf{E}}-\left|\nabla_{\mathbf{E}} u\right|(x)^{2}\left\langle X_{3} \nu(x), v(x)\right\rangle_{\mathbf{E}}
$$

Proof. By definition in $\mathbf{E}_{0}$

$$
\left\langle\nabla_{\mathbf{E}} X_{3} u, v\right\rangle_{\mathbf{E}}=\frac{1}{\left|\nabla_{\mathbf{E}} u\right|}\left(X_{1} X_{3} u X_{2} u-X_{2} X_{3} u X_{1} u\right)
$$

and using (7) we obtain

$$
\left\langle\nabla_{\mathbf{E}} X_{3} u, v\right\rangle_{\mathbf{E}}=\frac{1}{\left|\nabla_{\mathbf{E}} u\right|}\left[\left(X_{3} X_{1} u X_{2} u-X_{3} X_{2} u X_{1} u\right)+X_{4} u\left(X_{2} u-X_{1} u\right)\right] .
$$

Moreover, in $\mathbf{E}_{0}$,

$$
\left\langle X_{3} \nu, v\right\rangle_{\mathbf{E}}=\frac{1}{\left|\nabla_{\mathbf{E}} u\right|^{2}}\left(X_{3} X_{1} u X_{2} u-X_{3} X_{2} u X_{1} u\right) .
$$

Hence adding (37) and (38) we get the thesis.

Using Theorem 1.1 and Lemma 3.8 it immediately follows that

Corollary 3.9. Let $u \in C^{\infty}(\Omega)$ be a stable weak solution of (1). Then, for each $\eta \in C_{0}^{\infty}(\Omega)$

$$
\begin{aligned}
& \int_{\mathbf{E}_{0}}\left(\left|\nabla_{\mathbf{E}} u\right|^{2}\left\{\left(p+\frac{\left\langle(H u)^{T} \nu, v\right\rangle_{\mathbf{E}}}{\left|\nabla_{\mathbf{E}} u\right|}\right)^{2}+h^{2}\right\}+\left|\nabla_{\mathbf{E}} u\right|\left\langle\nabla_{\mathbf{E}} X_{3} u, v\right\rangle_{\mathbf{E}}\right. \\
& \left.+\left|\nabla_{\mathbf{E}} u\right|^{2}\left\langle X_{3} \nu, v\right\rangle_{\mathbf{E}}\right) \eta^{2} \leq \int_{\mathbf{E}}\left|\nabla_{\mathbf{E}} \eta\right|^{2}\left|\nabla_{\mathbf{E}} u\right|^{2} .
\end{aligned}
$$




\section{Some applications to entire stable solutions: geometric equations and non-existence results}

From now on, for every $x=\left(x_{1}, x_{2}, x_{3}, x_{4}\right) \in \mathbf{E}$, we denote by

$$
|x|:=\left(\left(x_{1}^{2}+x_{2}^{2}\right)^{6}+x_{3}^{6}+x_{4}^{4}\right)^{\frac{1}{12}}
$$

the standard gauge norm in $\mathbf{E}$ (see [7], [10]), and we denote by

$$
B(0, R):=\{x \in \mathbf{E}|| x \mid<R\}
$$

the gauge open ball centered at 0 of radius $R$.

The following Lemma is proved in [13].

Lemma 4.1. Let $g \in L_{\mathrm{loc}}^{\infty}\left(\mathbf{R}^{n},[0,+\infty)\right)$ and let $q>0$. Let also for any $\tau>0$,

$$
\eta(\tau):=\int_{B(0, \tau)} g(x) \mathrm{d} x
$$

Then for every $0<r<R$,

$$
\int_{B(0, R) \backslash B(0, r)} \frac{g(x)}{|x|^{q}} \mathrm{~d} x \leq q \int_{r}^{R} \frac{\eta(\tau)}{\tau^{q+1}} \mathrm{~d} \tau+\frac{1}{R^{q}} \eta(R) .
$$

Corollary 4.2. Let $u$ be a stable solution of $\Delta_{\mathbf{E}} u=f(u)$ in the whole of $\mathbf{E}$ with

$$
\mathcal{J} \leq 0 \text { in } \mathbf{E}_{0} \text {. }
$$

For any $\tau>0$ and any $x=\left(x_{1}, x_{2}, x_{3}, x_{4}\right) \in \mathbf{E}$, let us define

$$
\eta(\tau):=\int_{B(0, \tau)}\left|\nabla_{\mathbf{E}} u(x)\right|^{2} \mathrm{~d} x .
$$

If

$$
\liminf _{R \rightarrow \infty} \frac{\int_{\sqrt{R}}^{R} \frac{\eta(\tau)}{\tau^{3}} \mathrm{~d} \tau+\frac{\eta(R)}{R^{2}}}{(\log R)^{2}}=0
$$

then the level sets of $u$ in the proximity of noncharacteristic points are such that

$$
\operatorname{div}_{\mathbf{E}} \nu=0
$$

and on such sets the following equation holds

$$
p=-\frac{1}{\left|\nabla_{\mathbf{E}} u\right|}\langle H u v, \nu\rangle_{\mathbf{E}} .
$$

Proof. This is a modification of the proof of Corollary 3.2 of [13], where we take into account the more complicated algebraic calculations of the Engel group. Given $R>1$, we define

$$
\phi_{R}(x):= \begin{cases}1 & \text { if } x \in B(0, \sqrt{R}), \\ 2(\log R)^{-1} \log (R /|x|) & \text { if } x \in B(0, R) \backslash B(0, \sqrt{R}), \\ 0 & \text { if } x \in \mathbf{E} \backslash B(0, R) .\end{cases}
$$

We observe that

$$
\begin{aligned}
& X_{1}|x|^{12}=12\left(x_{1}^{2}+x_{2}^{2}\right)^{5} x_{1}-A x_{2}-2 x_{3} x_{4}^{3}, \quad \text { and } \\
& X_{2}|x|^{12}=12\left(x_{1}^{2}+x_{2}^{2}\right)^{5} x_{2}+A x_{1}-2 x_{3} x_{4}^{3}
\end{aligned}
$$


with $A:=3 x_{3}^{5}+(1 / 3)\left(x_{1}+x_{2}\right) x_{4}^{3}$. Since $\left|x_{1}\right| \leq|x|,\left|x_{2}\right| \leq|x|,\left|x_{3}\right| \leq|x|^{2}$ and $\left|x_{4}\right| \leq$ $|x|^{3}$, we conclude that $|A| \leq C_{1}|x|^{10}$ and so

$$
\left.\left.\left|\nabla_{\mathbf{E}}\right| x\right|^{12}\left|\leq C_{2}\right| x\right|^{11}
$$

for some $C_{1}, C_{2}>0$.

Notice also that, in $B(0, R) \backslash B(0, \sqrt{R})$,

$$
\phi_{R}(x)=C(R)-(1 / 6)(\log R)^{-1} \log |x|^{12}
$$

for some $C(R) \in \mathbf{R}$, thus

$$
\left|\nabla_{\mathbf{E}} \phi_{R}(x)\right|=\left.\left.(1 / 6)(\log R)^{-1}|x|^{-12}\left|\nabla_{\mathbf{E}}\right| x\right|^{12}\left|\leq C_{3}(\log R)^{-1}\right| x\right|^{-1}
$$

in $B(0, R) \backslash B(0, \sqrt{R})$ for some $C_{3}>0$. Therefore, by (41) and Theorem 1.1,

$$
\begin{aligned}
& \int_{\mathbf{E}_{0}}\left[\left|\nabla_{\mathbf{E}} u\right|^{2}\left\{\left(p+\frac{\left\langle(H u)^{T} \nu, v\right\rangle}{\left|\nabla_{\mathbf{E}} u\right|}\right)^{2}+h^{2}\right\}\right] \phi_{R}^{2} \\
& \leq \int_{\mathbf{E}}\left|\nabla_{\mathbf{E}} \phi_{R}\right|^{2}\left|\nabla_{\mathbf{E}} u\right|^{2} \leq C_{4}(\log R)^{-2} \int_{B(0, R) \backslash B(0, \sqrt{R})} \frac{\left|\nabla_{\mathbf{E}} u\right|^{2}}{|x|^{2}}
\end{aligned}
$$

for some $C_{4}>0$. On the other hand, by Lemma 4.1,

$$
\int_{B(0, R) \backslash B(0, \sqrt{R})} \frac{\left|\nabla_{\mathbf{E}} u\right|^{2}}{|x|^{2}} \leq 2 \int_{\sqrt{R}}^{R} \frac{\eta(\tau)}{\tau^{3}} \mathrm{~d} \tau+\frac{1}{R^{2}} \eta(R) .
$$

All in all,

$$
\begin{aligned}
& \int_{\mathbf{E}_{0}}\left[\left|\nabla_{\mathbf{E}} u\right|^{2}\left\{\left(p+\frac{\left\langle(H u)^{T} \nu, v\right\rangle}{\left|\nabla_{\mathbf{E}} u\right|}\right)^{2}+h^{2}\right\}\right] \phi_{R}^{2} \\
& \leq 2 C_{4}(\log R)^{-2}\left[\int_{\sqrt{R}}^{R} \frac{\eta(\tau)}{\tau^{3}} \mathrm{~d} \tau+\frac{1}{R^{2}} \eta(R)\right]
\end{aligned}
$$

Then the claim follows by sending $R \rightarrow \infty$, thanks to (43).

Remark 4.3. Recalling Lemma 3.8, we observe that (41) is implied by the following monotonicity conditions:

$$
\left\langle X_{3} \nu, v\right\rangle_{\mathbf{E}} \geq 0 \quad \text { and } \quad\left\langle\nabla_{\mathbf{E}} X_{3} u, v\right\rangle_{\mathbf{E}} \geq 0
$$

Remark 4.4. Condition (43) may be seen as a bound on the energy growth: for instance, it is satisfied if $\eta(R) / R^{2}$ stays bounded for large $R$, i.e., if the energy in $B(0, R)$ does not grow more than $R^{2}$. Of course, this is quite a strong assumption on the decay of $\nabla_{\mathbf{E}} u$ in the variables $\left(x_{3}, x_{4}\right)$ and it would be desirable to investigate in which way such condition may be weakened.

Remark 4.5. We stress that equations (44) and (45) may be seen as geometric equations along the level sets of the solution $u$. In particular, (44) may be stated as saying that the level set is a minimal surface for the Engel framework (in analogy with the Euclidean setting and in the terminology of [19]). Also, (45) is a prescription on the imaginary curvature $p$, in relation with the Hessian, the normal, and the tangent vectors. 
Remark 4.6. Let us observe that if $u$ is a solution of (1.1) that does not depend on $x_{3}$ and $x_{4}$ then $u$ satisfies $\Delta u=f(u)$, where $\Delta$ is the classical Euclidean Laplacian. Moreover, by [13, Remark 3.4], every bounded stable solution of (1.1) that does not depend on the last two coordinates and satisfies (43) has to be constant.

Theorem 4.7. There exists no stable solution of $\Delta_{\mathbf{E}} u=f(u)$ satisfying

(i) the zeros of $\ddot{f}$ (if any) are isolated;

(ii) $\left\{x \in \mathbf{E} \mid \nabla_{\mathbf{E}} u(x)=0\right\}=\varnothing$;

(iii) $u \in L^{\infty}(\mathbf{E})$;

(iv) $\left\langle X_{3} \nu, v\right\rangle_{\mathbf{E}} \geq 0$ in $E$;

(v) $\left\langle\nabla_{\mathbf{E}} X_{3} u, v\right\rangle_{\mathbf{E}} \geq 0$ in $E$;

(vi) the set $\left\{\left(X_{1} u+X_{2} u\right)=0\right\}$ has zero Lebesgue measure;

(vii) $\liminf _{R \rightarrow \infty} \frac{\int_{\sqrt{R}}^{R} \frac{\eta(\tau)}{\tau^{3}} \mathrm{~d} \tau+\frac{\eta(R)}{R^{2}}}{(\log R)^{2}}=0$, where $\eta$ is as in Corollary 4.2.

Proof. We shall prove that if $u \in C^{3}(\mathbf{E})$ is a stable solution of (1) which satisfies (i), (ii), (iii), (iv), (v), (vi) and (vii), then $X_{3} u=0$ and $X_{4} u=0$. Hence $u$ is independent of $x_{3}$ and $x_{4}$ and this gives the desired result. By (iii) and [8, Th. 2.10] we have

$$
\left|\nabla_{\mathbf{E}} u\right| \in L^{\infty}(\mathbf{E})
$$

We claim that

$$
X_{3} u=0 \text { in } \mathbf{E} .
$$

To this end, we argue by contradiction, supposing that there exists $Q \in \mathbf{E}$ such that

$$
X_{3} u(Q) \neq 0 .
$$

Thus we consider the following Cauchy problem

$$
\left\{\begin{array}{l}
\phi^{\prime}(s)=v(\phi(s)), \\
\phi(0)=Q
\end{array}\right.
$$

where $v$ is as in (3). By (ii) and the fact that $|v|=1$ it follows that the solution exists and it is defined for any $s \in \mathbf{R}$. Moreover, by (ii)

$$
u(\phi(s))^{\prime}=\left\langle\nabla_{\mathbf{E}} u(\phi(s)), \phi^{\prime}(s)\right\rangle_{\mathbf{E}}=\left|\nabla_{\mathbf{E}}(\phi(s))\right|\langle\nu(\phi(s)), v(\phi(s))\rangle_{\mathbf{E}}=0 \quad \forall s \in \mathbf{R},
$$

that is, $\phi$ lies on the level set of $u$, namely

$$
\phi(s) \in\{x \in \mathbf{E} \mid u(x)=u(Q)\} \quad \forall s \in \mathbf{R} .
$$

Furthermore,

$$
\left|\nabla_{\mathbf{E}} u(\phi(s))\right|^{\prime}=\left\langle\nabla_{\mathbf{E}}\left|\nabla_{\mathbf{E}} u\right|(\phi(s)), \phi(s)^{\prime}\right\rangle_{\mathbf{E}} \quad \forall s \in \mathbf{R},
$$

and by (14) (applied here with $\eta \equiv 1$ ) and Corollary 4.2 (recall also Remark 4.3) we get

$$
\begin{aligned}
\left|\nabla_{\mathbf{E}} u(\phi(s))\right|^{\prime} & =\frac{1}{\left|\nabla_{\mathbf{E}} u(\phi(s))\right|}\left\langle(H u)^{T} \nabla_{\mathbf{E}} u(\phi(s)), v(\phi(s))\right\rangle_{\mathbf{E}} \\
& =\langle\nu(\phi(s)),(H u) v(\phi(s))\rangle_{\mathbf{E}}=-\left|\nabla_{\mathbf{E}} u(\phi(s))\right| p(\phi(s)) \\
& =X_{3} u(\phi(s)) \quad \forall s \in \mathbf{R},
\end{aligned}
$$


which, via (48), implies

$$
\left|\nabla_{\mathbf{E}} u(\phi(s))\right|_{\mid s=0}^{\prime} \neq 0 .
$$

From (49) we deduce

$$
\begin{aligned}
\left|\nabla_{\mathbf{E}} u(\phi(s))\right|^{\prime \prime} & =\left(X_{3} u(\phi(s))\right)^{\prime}=\left\langle\nabla_{\mathbf{E}} X_{3} u(\phi(s)), \phi^{\prime}(s)\right\rangle_{\mathbf{E}} \\
& =\left\langle\nabla_{\mathbf{E}} X_{3} u(\phi(s)), v(\phi(s))\right\rangle_{\mathbf{E}} \quad \forall s \in \mathbf{R},
\end{aligned}
$$

and by (iv) we deduce also that

$$
\left|\nabla_{\mathbf{E}} u(\phi(s))\right|^{\prime \prime} \geq 0 \quad \forall s \in \mathbf{R} .
$$

Therefore, defining $\Phi: \mathbf{R} \rightarrow \mathbf{R}$ by

$$
\Phi(s):=\left|\nabla_{\mathbf{E}} u(\phi(s))\right|-\left|\nabla_{\mathbf{E}} u(Q)\right|
$$

we have that $\Phi \in C^{2}(\mathbf{R}), \Phi(0)=0, \Phi^{\prime}(s) \neq 0 \forall s \in \mathbf{R}$ and $\Phi^{\prime \prime}(s) \geq 0 \forall s \in \mathbf{R}$, thanks to $(50)$ and (52). It follows that

$$
\sup _{\mathbf{R}} \Phi=+\infty
$$

but this is in contradiction with (46), hence (47) is established.

Now we claim that

$$
X_{4} u=0 .
$$

By Corollary 3.4, we have

$$
\Delta_{\mathbf{E}} X_{3} u-2 X_{4} X_{1} u-2 X_{4} X_{2} u=X_{3} \Delta_{\mathbf{E}} u=X_{3}(f(u))=\dot{f}(u) X_{3} u
$$

and so by (47) it follows that

$$
X_{4}\left(X_{1} u+X_{2} u\right)=0 .
$$

Moreover, by Corollary 3.4 and (47),

$$
\begin{aligned}
\Delta_{\mathbf{E}} X_{1} u-X_{4} u & =\Delta_{\mathbf{E}} X_{1} u-2 X_{4} u+X_{4} u \\
& =\Delta_{\mathbf{E}} X_{1} u-2\left(X_{2} X_{3}-X_{3} X_{2}\right) u+X_{4} u \\
& =\Delta_{\mathbf{E}} X_{1} u+2 X_{3} X_{2} u+X_{4} u=\dot{f}(u) X_{1} u
\end{aligned}
$$

and

$$
\Delta_{\mathbf{E}} X_{2} u+X_{4} u=\Delta_{\mathbf{E}} X_{2} u-2 X_{1} X_{3} u+X_{4} u=\dot{f}(u) X_{2} u .
$$

By adding (55) and (56) we obtain

$$
\Delta_{\mathbf{E}}\left(X_{1} u+X_{2} u\right)=\dot{f}(u)\left(X_{1} u+X_{2} u\right)
$$

and so, by Lemma 3.3,

$$
\begin{aligned}
\Delta_{\mathbf{E}} X_{4}\left(X_{1} u+X_{2} u\right) & =X_{4} \Delta_{\mathbf{E}}\left(X_{1} u+X_{2} u\right)=X_{4}\left(\dot{f}(u)\left(X_{1} u+X_{2} u\right)\right) \\
& =\ddot{f}(u) X_{4} u\left(X_{1} u+X_{2} u\right)+\dot{f}(u) X_{4}\left(X_{1} u+X_{2} u\right) .
\end{aligned}
$$

Accordingly, using (58) and (54), we conclude that

$$
\ddot{f}(u) X_{4} u\left(X_{1} u+X_{2} u\right)=0 \quad \text { in } \mathbf{E} .
$$

Hence, by (vi)

$$
\ddot{f}(u) X_{4} u=0 \quad \text { almost everywhere in } \mathbf{E}
$$


and so, by continuity,

$$
\ddot{f}(u) X_{4} u=0 \quad \text { everywhere in } \mathbf{E} \text {. }
$$

This implies that (53) holds at any point of the open set $G:=\{x \in \mathbf{E} \mid \ddot{f}(u(x)) \neq 0\}$. So, by continuity, (53) holds at any point of its closure $\bar{G}$.

We show that (53) also holds at points of $\mathbf{E} \backslash \bar{G}$ (if any). For this, let us take $x_{o} \in$ $\mathbf{E} \backslash \bar{G}$. Since the latter is an open set, there exists an open neighborhood $V$ such that

$$
x_{o} \in V \subseteq(\mathbf{E} \backslash \bar{G}) \subseteq \mathbf{E} \backslash G=\{x \in \mathbf{E} \mid \ddot{f}(u(x))=0\} .
$$

In particular, $\ddot{f}(u(x))=0$ for any $x \in V$. Thus, by (i), $u(x)$ must be constant for any $x \in V$. Therefore, $X_{4} u(x)=0$ for any $x \in V$, and, in particular, $X_{4} u\left(x_{o}\right)=0$.

This shows that (53) holds at points of $\mathbf{E} \backslash \bar{G}$ too, and so the proof of (53) is completed.

Now, by (47) and (53), we conclude that $u$ does not depend on $x_{3}$ and $x_{4}$ and by Remark 4.6 we conclude that $u$ is constant but this is impossible by (ii), which proves Theorem 4.7 .

Remark 4.8. Of course, we do not believe that our Theorem 4.7 is optimal: we just consider it a first attempt towards the understanding of semilinear equations in the Engel framework and, as far as we know, this is the first non-existence result in this setting. We think it would be interesting to develop a stronger theory and possibly to drop some structural assumptions in Theorem 4.7.

Acknowledgments. We thank the referee for her or his interesting comments. The second author was supported by the MIUR National Research Project Nonlinear Elliptic Problems in the Study of Vortices and Related Topics, the FIRB project A\&B Analysis and Beyond and the ERC project $\epsilon$ Elliptic Pde's and Symmetry of Interfaces and Layers for Odd Nonlinearities.

\section{References}

[1] Arcozzi, N., and F. Ferrari: Metric normal and distance function in the Heisenberg group. - Math. Z. 256, 2007, 661-684.

[2] Arcozzi, N., and F. Ferrari: The Hessian of the distance from a surface in the Heisenberg group. - Ann. Acad. Sci. Fenn. Math. 33, 2008, 35-63.

[3] Arcozzi, N., F. Ferrari, and F. Montefalcone: $C C$-distance and metric normal of smooth hypersurfaces in sub-Riemannian Carnot groups. - arXiv:0910.5648v1.

[4] Birindelli, I., F. Ferrari, and E. Valdinoci: Semilinear PDEs in the Heisenberg group: the role of the right invariant vector fields. - Nonlinear Anal. 72, 2010, 987-997.

[5] Birindelli, I., and E. LAnCOnelli: A negative answer to a one-dimensional symmetry problem in the Heisenberg group. - Calc. Var. Partial Differential Equations 18, 2003, 357-372.

[6] Birindelli, I., and J. Prajapat: Monotonicity results for nilpotent stratified groups. - Pacific J. Math. 204, 2002, 1-17.

[7] Bonfiglioli, A., E. Lanconelli, and F. Uguzzoni: Stratified Lie groups and potential theory for their sub-Laplacians. - Springer Monogr. Math., Springer, Berlin, 2007.

[8] Capogna, L., and Q. Han: Pointwise Shauder estimates for second order linear equations in Carnot groups. - In: Harmonic analysis at Mount Holyoke (South Hadley, MA, 2001), Contemp. Math. 320, 2003, 45-69. 
[9] Chow, W. L.: Über Systeme von linearen partiellen Differentialgleichungen erster Ordung. Math. Ann. 117, 1939, 98-105.

[10] Danielli, D., N. Garofalo, and D. M. Nhieu: Sub-Riemannian calculus on hypersurfaces in Carnot groups. - Adv. Math. 215, 2007, 292-378.

[11] Farina, A.: Habilitation diriger la recherche. - 2002.

[12] Farina, A., B. Sciunzi, and E. Valdinoci: Bernstein and De Giorgi type problems: new results via a geometric approach. - Ann. Sc. Norm. Super. Pisa Cl. Sci. (5) 7, 2008, 741-791.

[13] Ferrari, F., and E. Valdinoci: A geometric inequality in the Heisenberg group and its applications to stable solutions of semilinear problems. - Math. Ann. 343, 2009, 351-370.

[14] Ferrari, F., and E. Valdinoci: Geometric PDEs in the Grushin plane: weighted inequalities and flatness of level sets. - Int. Math. Res. Not. IMRN 22, 2009, 4232-4270.

[15] Ferrari, F., and E. Valdinoci: Some weighted Poincaré inequalities. - Indiana Univ. Math. J. 58, 2009, 1619-1637.

[16] Franchi, B., R. Serapioni, and F. Serra Cassano: Meyer-Serrin type theorems and relaxation of variational integrals depending on vector fields. - Houston J. Math. 22, 1996, 859-890.

[17] Garofalo, N., and E. Lanconelli: Existence and nonexistence results for semilinear equations on the Heisenberg group - Indiana Univ. Math. J. 41, 1992, 71-98.

[18] Hörmander, L.:, Hypoelliptic second order differential equations. - Acta Math. 119, 1967, $147-171$.

[19] Monti, R., and F. Serra Cassano: Surface measures in Carnot-Carathéodory spaces. Calc. Var. Partial Differential Equations 13, 2001, 339-376.

[20] Pauls, S. D.: Minimal surfaces in the Heisenberg group. - Geom. Dedicata 104, 2004, 201-231.

[21] Sternberg, P., and K. Zumbrun: A Poincaré inequality with applications to volumeconstrained area-minimizing surfaces. - J. Reine Angew. Math. 503, 1998, 63-85.

[22] Sternberg, P., and K. Zumbrun: Connectivity of phase boundaries in strictly convex domains. - Arch. Ration. Mech. Anal. 141, 1998, 375-400.

Received 1 July 2011 